\title{
CONSCiOUSNESS, Situations, AND THE MEASUREMENT PROBLEM OF QUANTUM MECHANICS
}

\author{
Michel Bitbol \\ CREA, CNRS / Ecole Polytechnique, 1, rue Descartes, 75005 Paris, France
}

NEUROQUANTOLOGY, 6, 203-213, 2008

\begin{abstract}
:
There are two versions of the putative connection between consciousness and the measurement problem of quantum mechanics : consciousness as the cause of state vector reduction, and state vector reduction as the physical basis of consciousness. In this article, these controversial ideas are neither accepted uncritically, nor rejected from the outset in the name of some prejudice about objective knowledge. Instead, their origin is sought in our most cherished (but disputable) beliefs about the place of mind and consciousness in the world. It is first pointed out that these common beliefs about mind and consciousness arise from reification of situated first-person experience. Then, situatedness is shown to be a constitutive part of any exhaustive treatment of quantum measurements. It turns out that the alleged connection between consciousness and the measurement problem is a symptom of (i) the ineliminability of our being situated from the end-product of science, and (ii) our difficulty to express correctly this being situated.
\end{abstract}

Keywords : Quantum mechanics, Consciousness, Situatedness, Perspective, Phenomenology, Naturalization, Transcendental epistemology, Measurement problem, Objectivity, Wave-packet collapse

\section{Introduction}

The story started during a discussion session of the Solvay conference held in Brussels in 1927 (See G. Bacciagaluppi \& A. Valentini [2008]). Dirac was trying to make sense of the contrast between the wave function of quantum mechanics, which presents a list of possible experimental results together with their probability amplitudes, and the fact that we just obtain one of these results in any given particular experiment. He then invoked a mysterious "choice of nature" which was supposed to privilege one result in advance. But Heisenberg did not agree at all. He insisted that the idea that nature had already chosen a result before the experiment is completed, could but lead to incorrect probabilistic predictions. He thus suggested that the choice, if any, is performed at the very end of the experimental process, when an observer comes into play. The alleged choice which yields state vector reduction is not the choice of nature, according to Heisenberg, but "the choice of the observer".

During the following years, Heisenberg's proposal was eagerly criticized by some physicists, whereas others rather tried to make it more specific. Among the latter, we find von Neumann [1955], who invoked the "abstract self" of the observer in order to account for state vector reduction; Schrödinger [1935] who spoke half-ironically of a "mental act"; London and Bauer [1939] (see S. French [2002]) who toyed with the idea of solipsism but finally insisted on the crucial human feature of "introspective power"; and last but not least Wigner [1967] who advocated the central role of "consciousness" in state vector reduction. There were also other authors (like R. Penrose [1994], S. Hameroff [1996], and H. Stapp [2007]) who put the problem upside down and tried to solve the "hard problem" of the putative material origin of consciousness by grounding it on state vector reduction taken as a fact of nature.

In this article, my purpose is neither to accept these ideas uncritically, nor to reject them from the outset in the name of a narrow conception of objectivity, but rather to find their rationale and to analyze the reasons of their having been felt so compelling by so many great 
thinkers. The central reason for what I consider as a common misunderstanding about the relation between consciousness and the measurement problem of quantum mechanics is reification of first-person experience (Bitbol [2000a], [2000b], [2002], [2008]).

\section{Adjectives and nouns : about consciousness}

Let me then begin with some definitions. "Consciousness" is one of those words which arose from a trend towards substantivization of common adjectives. Here, the adjective is "conscious". Its meaning is established by studying the boundary between the situations where it can be used to qualify a person (including oneself) or an action, and the situations like sleep, fainting, anesthaesia, somnambulism etc., where one should rather drop it or replace it by the adjective "unconscious". In the case of one's own normal waking state, the adjective "conscious" does little more than a sort of verbal gesticulation towards "that which goes without saying" (Wittgenstein [1982]), namely the mere fact that there is lived embodied experience rather than nothing. But once the threshold of substantivization has been crossed, the temptation, which was repeatedly denounced by Wittgenstein, is to look for some substance which could play the role of a referent of the substantive. Gilbert Ryle [1949] accordingly argued that the noun "consciousness" has the cartesian doctrine of the thinking substance as its most natural counterpart. Consciousness is commonly declared to be something of the mind as opposed to the body. It is taken as the constant self-awareness of the operations of the mind. If systematically directed towards some mental acts or mental contents, consciousness is then said to give rise to introspection. And, through consciousness and introspection, it is thought that mind has a privileged access to its own doings, as opposed to the unprivileged access which one may obtain by studying behaviour and speech.

We thus see that the correlates of this traditional conception of consciousness cover the whole field of notions which was invoked in relation to the measurement problem of quantum mechanics. Mind as the most specific part of the self; mental acts; introspective power; and privileged access which can be used as an argument for solipsism. Here again, I don't want to discard but rather to understand. Why did this doctrine of consiousness came out, and why did it stay so popular for so many centuries including our own, in spite of its having been cogently criticized again and again?

Fortunately, it is not difficult to find an answer to the last two questions. Cartesian dualism of thinking substance and extended substance is a straightforward (some would say too straightforward) expression of a basic functional duality which often arises in speech when several persons do not immediately reach agreement about something.

To understand what is this "functional duality", let us consider a few examples. With a caricatural paradigmatic sentence such as "the cat is on the mat", no special precaution is needed, for every person who is in the same room as the celebrated cat is very likely to agree about its being on the mat. But think of another situation. John says "this tree is green", whereas Peter, showing the same tree, says "this tree is red". The most immediate way by which the two protagonists may deal with this flat contradiction is to have recourse to personal pronouns. John will correct his first sentence by saying " $I$ see this tree green" and Peter by saying " $I$ see this tree red". The contradiction is defused by restricting the field of application of each one of its term to a particular self using the personal pronoun "I". Later on, each one of the two protagonists can control the whole procedure by using two personal pronouns instead of one, thereby reaching a kind of pronominal agreement. For instance, John could assert: "I see this tree green and you see this same tree red" while Peter can perfectly agree with John on the proposition expressed by his sentence, and manifest his agreement by just inverting the place of the personal pronouns: "Yes, it's true: You see this tree green and I see this tree red".

The first move allowing several persons to reach agreement on something thus consists in separating clearly what depends on a particular situation or a personal state from what is essentially independent of this situation or state. In practice, as we have just seen, this is done by introducing personal pronouns in sentences in the first case, and dropping them in the second 
case. Such a grammatical distinction between particular situations and universal states is just what I meant by "functional duality".

Now, one generally does not stop at this stage. The second move towards mutual agreement consists in trying to identify the reason of the difference of judgment, in such a way that everybody may agree directly about this reason even without making use of personal pronouns. For instance, John and Peter may agree that the reason of their not seeing the same colour is that Peter's retinal cones lack some appropriate molecules enabling most human beings to distinguish between green and red. The reason, in a word, is that Peter is Daltonian. They can even agree on the fact that the wavelength of the light reflected by the leaves of the tree is about 530 nanometers. This is nice, and this is exactly what science is aimed at: objectivity. But beware! The impersonal agreement between John, Peter and many others about Peter's genetic anomaly and about the wavelength of reflected light is only a second order agreement. It is agreement about the reason of the initial disagreement. It does not alleviate, let alone eliminate, the difference of personal judgment about the colour of the tree. And the latter, here again, can only be tackled by a proper use of personal pronouns.

However, the pattern of impersonal sentences and impersonal agreement is so dominant, that one may make a last and desperate attempt to dispense with personal pronouns and other indexicals when a difference of judgment is at stake. It consists in projecting the particularity of a first-person situation or viewpoint onto a third-person entity called "the mind" or "the thinking substance". The initial difference of personal judgment is then expressed in terms of a difference of mental content privately accessible by consciousness.

Hence the paradox of an entity (the mind) built on the model of public objects in order to embody privacy.

It is especially interesting for our inquiry that Schrödinger [1995], one of the creators of quantum mechanics, made essentially the same point in his William James lectures of 1954, though with a slightly different philosophical outlook, “(...) How do red and yellow, sweet and hot come in at all? Once we have removed them from our 'objective world' we are at a desperate loss to restore them. We cannot remove them entirely, because they are there, we cannot argue them away. So we have to give them a living space, and we invent a new realm for them, the mind, saying that this is where they are (...)".

We have now progressed sufficiently to see our initial question in a very different light. If Mind is but a limiting projection of the particularity of viewpoints or situations, and if primary Consciousness is but an expression of experiential access from any viewpoint, then the question "has consciousness anything to do with quantum mechanics?" itself appears as a projection, or a metaphorical expression, of another question. This other question is the following: "Does quantum mechanics impose, more strongly than any other physical theory, taking into account the articulation between particular viewpoints and universal statements?".

As it will soon be clear, the answer to the latter question is positive. But of course, a positive answer to that question does not yield the same paradoxical consequences as a positive answer to our original question. No spurious effect of mind on matter to be feared (or to be greeted), but only the following plain remark. Everyday speech maintains a functional duality between particular and universal statements, between first person and third person discourse, between indexical and non-indexical reference. In classical physics, it proved quite easy to sweep the first element of this functional duality under the carpet, but this is no longer the case in quantum mechanics.

\section{A thought experiment about probability and situations (classical version)}

To show this, I shall make use of a very simple thought experiment, in two versions. A very familiar version, and another version slightly modified in order to take into account the special constraints to which quantum physics is submitted.

Let us imagine that we are sitting in an office separated from a laboratory by a wall and a door. Inside the laboratory, which is carefully isolated from the rest of the world, some experiments are automatically carried out. Their result will be accessible to us well after their 
completion, at the instant when we open the door which separates us from the spot where they took place. The first situation I will consider is a single event of tossing a coin, performed by a mechanical device generating random accelerations. Having very little knowledge about the coin and the mechanical device, the best kind of prediction we can make about the final position of the coin on the floor is a probabilistic one. We shall then define a probability function which associates a real number ranging between 0 and 1 to each one of the two events (head=h or tail=t). By using the only a priori information we have about the coin, namely its being essentially symmetrical, the most likely estimate of the two probabilities is:

$$
\mathrm{P}(\mathrm{h})=1 / 2 \text { and } \mathrm{P}(\mathrm{t})=1 / 2
$$

Of course, nothing prevents us from testing this estimate by comparing it to the frequency of occurrence of heads and tails during a large number of tosses. But I will not insist on this point, and rather concentrate on some refinements of the original thought experiment.

Let us suppose that tosses occur in the presence of an apparatus made of a video camera connected to a computer with a software allowing it to identify shapes. As soon as the coin is lying on the floor, the computer switches on and it translates the picture coming from the camera into a chain of characters (either H.E.A.D. or T.A.I.L.) printed on its screen. If both the camera and the computer are reliable, we can be sure that we shall find H.E.A.D. on the screen if the coin is on head, and conversely T.A.I.L. on the screen if the coin is on tail.

We can write this:

$$
\begin{array}{ll}
\text { P(H.E.A.D. if } h)=1 & \text { P(T.A.I.L. if } t)=1 \\
\text { P(H.E.A.D. if } t)=0 & \text { P(T.A.I.L. if } h)=0
\end{array}
$$

This kind of interaction between an object and an apparatus, translating accurately an event into a chain of characters, is called a "good measurement". This being granted, we can easily calculate the probability of our seing "H.E.A.D." on the computer screen when we enter into the laboratory:

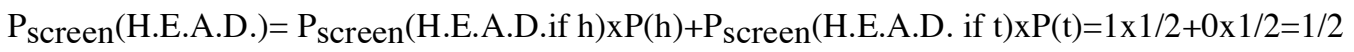

In other words, during a good measurement, the probability function of a certain result printed on the screen is exactly identical to that of the event itself.

Let us now add some embellishments suggested by the history of quantum mechanics (but still in a classical context).

Firstly we may add a cat, a hammer, and a flask of poison. The computer makes the hammer fall on the flask of poison if it has "H.E.A.D." on its screen, and it does nothing if it has "T.A.I.L." on its screen. By the same probabilistic reasoning as previously, we must admit that the probability of finding a dead cat when we open the door is exactly equal to the probability of the coin's falling on head, whereas the probability of finding a cat alive is exactly equal to the probability of the coin's falling on tail. We write this:

$\mathrm{P}_{\text {cat }}($ Dead $)=\mathrm{P}(\mathrm{h})=1 / 2$ and $\mathrm{P}_{\text {cat }}($ Alive $)=\mathrm{P}(\mathrm{t})=1 / 2$

Secondly, nothing prevents us from sending a human colleague in the laboratory (with a gas mask!). With the same probabilistic reasoning as previously, we can say that the probability for her answering us "I have seen the coin on tail, the chain of characters T.A.I.L. on the screen, and the cat alive" if we ask her, is exactly equal to the probability that the coin is on tail. And the same for head. This writes:

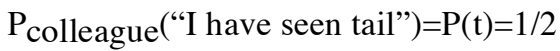

and Pcolleague("I have seen head") $=\mathrm{P}(\mathrm{h})=1 / 2$ 
Thus, our having introduced an apparatus, a cat and a colleague in the laboratory did not change anything to our initial estimate of the probabilities. This initial estimate has only been propagated from one link of the measurement chain to the next.

Everything changes, of course, when WE who made the probabilistic evaluation come into play. Everything changes when WE enter into the laboratory and become aware of what happened, by observing successively the coin, the screen and the cat, and then by asking our colleague what she noticed. Indeed, as soon as WE know what has occurred (for instance that the coin has fallen on tail etc.) the best prediction WE can make about the result we would find if we entered a second time in the laboratory in order to check the result is no longer:

$\mathrm{P}(\mathrm{t})=\mathrm{P}_{\text {screen }}($ T.A.I.L. $)=\mathrm{P}_{\text {cat }}(\mathrm{A})=\mathrm{P}_{\text {colleague }}$ ("I have seen tail” $)=1 / 2$

but:

$\mathrm{P}(\mathrm{t})=\mathrm{P}_{\text {screen }}($ T.A.I.L. $)=\mathrm{P}_{\text {cat }}(\mathrm{A})=\mathrm{P}_{\text {colleague }}$ (“I have seen tail”) $=1$

The probability function of each element of the measurement chain thus jumps suddenly from $1 / 2$ to 1 when WE become aware of the result. We shall call this sudden jump "the reduction of the probability function".

Let us examine this "reduction" more closely, for, except for a little but quite important nuance that I will discuss in due course, it is the very point which motivated the introduction of the concept of "consciousness" in the discourse of some quantum physicists.

(1) In the previous thought experiment, WE are the only ones able to reduce the probability function P. The observer sent before us in the lab. could not do that. Have we thus fallen into a sort of collective solipsism (namely, the solipsism of the persons who stayed in the office during the time the experiment was going on in the lab.)? Nobody would dare making such an accusation against us, not even the observer sent in the lab. For, after all, everybody realizes that the probability functions $\mathrm{P}, \mathrm{P}_{\text {Screen }}, \mathrm{P}_{\text {cat }}$, and $\mathrm{P}_{\text {colleague, }}$ were defined as the best possible estimate of the likelihood WE have to find the measurement chain in one of its two accessible states, taking into account OUR particular situation and OUR initial information. It is thus by no means surprising that the only people able to reduce THESE functions of probability are just the people who are in THIS particular situation and who have THIS initial information. The particular relation between US and THESE probability functions is due to the trivial fact that they were defined from the outset by explicit reference to US. If the observer who penetrated in the lab. before us had defined his own probability functions $\mathrm{P}^{*}$, the reduction of THIS LATTER function would have occurred at the instant of HIS becoming aware of the outcome of the experiment.

(2) These probability functions, which were initially construed as a tool of prediction about the result of a toss which had not yet occurred, are also the best instrument WE have for describing the situation in the lab. between the moment when the toss of the coin has occurred and the moment we penetrate in it. In view of our uncertainty, nothing prevents US from considering the probability functions $\mathrm{P}$ as OUR best available representation of the "state" of the measurement chain during this period. But remember the probability functions $\mathrm{P}$ and $\mathrm{P}^{*}$ are only "reduced" at the moment when one human observer becomes aware of the result of the experiment. Do we then have to say that consciousness (OUR consciousness or HIS consciousness, according to the probability function $\mathrm{P}$ or $\mathrm{P}^{*}$ one considers), has an effect on the state of the material object? Here again (in this classical version of the thought experiment), I think nobody would accept this. Everybody here realizes that the description of the state of the measurement chain as it is afforded by a probability function is only valid for a certain group of observers. It has no other role than expressing the partial ignorance of THIS group, about what has actually occurred in the lab. One has thus no reason to consider that the reduction of the probability function is the sign of an alteration of the material content of the lab.

In this classical case, the coins, the computers, the cats and the colleagues do what they are expected to do, namely falling on one side, printing a result, living or dying, and telling a result. In short, they acquire determinations which are proper to them. As for consciousnesses, they do what they were invented for, namely "becoming aware" of an experimental situation which 
occurred independently of them. The objects acquire properties, and the consciousnesses do nothing more ... than being conscious of these properties. This strict distribution of roles between mental and physical entities is in perfect agreement with cartesian dualism.

\section{A thought experiment about probability and situations (quantum version)}

The former remarks sound so obvious that an innocent (but clever) thinker may be surprised to discover that they do not apply to quantum probabilities.

But let us avoid precipitation. Before discussing the distinctive features of quantum probabilities, we still have to stress a very important feature of classical probabilities.

Let us focus our attention on the motion of the coin between its toss by the mechanical device and its fall on the floor. During this period, the coin can spin either clockwise (C) or counterclockwise (CO). Even if nothing or nobody records this direction of rotation, it is still possible to decompose the string of events in two steps: rotation (C or $\mathrm{CO}$ ) and fall (Head or Tail). Let us concentrate on the strings of events whose end is Head. There are two such strings: $(\mathrm{C}, \mathrm{h})$ and $(\mathrm{CO}, \mathrm{h})$. The coin can follow either the sequence $(\mathrm{C}, \mathrm{h})$ or the sequence $(\mathrm{CO}, \mathrm{h})$, but no mixture of them. This is called a disjunction. In such a case, the classical theory of probability indicates that the probability of obtaining "Head" is equal to the probability that the coin first rotates clockwise and then falls on head, plus the probability that the coin first rotates counterclockwise and then falls on head:

$$
\mathrm{P}(\mathrm{h})=\mathrm{P}(\mathrm{C}, \mathrm{h})+\mathrm{P}(\mathrm{CO}, \mathrm{h})
$$

It is precisely this formula that quantum probabilities violate. Of course, in order to adapt our vocabulary to that of physicists, it would be better to modify the first steps of our thought experiment. The toss of a coin should for instance be replaced by the emission of spin $1 / 2$ particles, the video camera by a Stern and Gerlach device, the position of a coin (head or tail) by the two eigenvalues $+1 / 2$ and $-1 / 2$ of the observable $S_{z}$ (Z-component of the spin), and finally the direction of rotation of the coin before it falls by the eigenvalues $+1 / 2$ and $-1 / 2$ of the observable $S_{x}$ (X-component of the spin).

However, since my purpose is to clarify ideas rather than going into technical details, I will keep on with coins and video cameras, endowing them with very strange features, very different at any rate from those of their familiar counterparts. Physicists are allowed to back to the former quantum concepts if they wish to think in terms of particles rather than in terms of "strange coins".

Let us then imagine the following: from now on, the coins are unable to adopt any definite position head or tail by themselves. But their random and uncontrollable interaction with the video camera can force the computer to print one of the two strings of characters "H.E.A.D." or "T.A.I.L." on its screen. In this case, of course, we cannot any longer ascertain that the whole process is a good measurement by just comparing the string of characters on the computer screen with the position that the coin has adopted independently of the measurement apparatus ; for, according to our assumption, there is no such independent position. The only criterion still available to ensure that the printing process is not just an artifact, but a true "measurement", then consists in repeating the video recording immediately after, with the same coin but with another camera, and seeing that the computer prints exactly the same string of characters. As Schrödinger underlined in his 1935 cat's paper, the ultimate criterion of a true measurement is the reproducibility of its result.

Now, what can we tell about the strange coin, between its throwing and its interaction with the camera? Just that it has a probability $1 / 2$ of triggering the printing of "H.E.A.D." on the screen of the computer and a probability $1 / 2$ of triggering the printing of "T.A.I.L." on the screen. At first sight, the situation looks very much like the one we described for ordinary coins. The instrument of prediction is identical in both situations: it is the theory of probabilities. And furthermore, in both situations, the function of probability $\mathrm{P}$ is the best description we can give of the state of the coin before its interaction with the camera. However a closer examination 
would show that something essential have changed from the classical to the quantum case. In the original (classical) case, nothing prevented one from thinking that the probabilities just expressed our ignorance about the position (head or tail) that the coin would adopt or had already adopted by itself. But in the second (quantum) case, this interpretation of probabilities becomes simply inacceptable. Indeed, the probabilities are here only defined relative to a possible interaction with a video camera. The position of the coin is no longer a property defined in absolute, but only an observable, defined by a relation. The difference may look quite abstract, but it is easy to show that it is not a purely intellectual one by bringing about some of its experimental consequences. In order to do so, we need to consider more than only one observable. For instance we may consider the observable direction of rotation of the coin, in addition to the observable position.

We mentioned previously that, according to the classical theory of probabilities, the probability that head obtains is equal to the probability that the coin rotates clockwise and falls on head plus the probability that it rotates counterclockwise and falls on head. This rule of addition expressed that the two directions of rotation were mutually exclusive. The coins which rotate clockwise do not rotate counterclockwise and vice-versa. But in the present case, the direction of rotation cannot be considered as a property that the coin has by itself. It is only defined relative to the possible interaction of the coin with an apparatus able to detect rotation; it is only an observable. Nothing enables one to make calculations as if the coins which rotate clockwise did not rotate counterclockwise and conversely, for the coins do not "rotate" properly speaking in one direction or another; they are waiting to interact with an apparatus able to translate this interaction into a string of characters "C." and "C.O.". The appropriate theory of probabilities thus has to take into account a certain amount of "wavering" of the coins as to the direction of their own rotation between the throwing and the measurement of their final position. This is done simply by adding a term $\mathrm{W}$ of "wavering" to the classical formula:

P(H.E.A.D.)=P(H.E.A.D.,C.)+P(H.E.A.D., C.O.)+W(H.E.A.D.,C,C.O.)

This formula is experimentally correct (for atoms or elementary particles, not ordinary coins!). And since the appropriate term $\mathrm{W}$ is exactly identical to the term of interference between two waves whose amplitudes A(H.E.A.D., C) and A(H.E.A.D., C.O.) are (roughly) the square root of P(H.E.A.D., C) and P(H.E.A.D., C.O.), everything goes as if we were dealing with waves rather than with the small material bodies which are our usual representation of atoms and particles. This is exactly the reason why the quantum mechanical description of the state of objects makes use of "wave functions" rather than "functions of probability". This deep-lying connection between the contextual character of observables, and the wave-like form of probability distributions was demonstrated mathematically by P. Destouches-Février [1956].

Let us recapitulate: for the objects which fall into the specific range of validity of quantum mechanics, the probabilities can by no means be considered as an expression of our ignorance about their properties. The probabilities rather express an a priori indeterminacy of the interaction between the objects and the relevant measuring apparatus; and this indeterminacy in turn manifests itself by wavelike effects.

This being granted, it seems however obvious that as soon as the coin and the camera (or more generally the object and the apparatus) have interacted, everything must change. It seems obvious that the computer connected to the camera now has a genuine property, namely its having H.E.A.D. or T.A.I.L. written on its screen, and that the function of probability of our finding a certain result on the screen when we enter into the lab. can thus be interpreted from now on as just an expression of our ignorance about the effective property of the computer screen. Unfortunately, things are not so simple. One should not forget that the apparatuses, the video cameras and the computers are made of atoms, namely of components which are just as strange as the "strange coin" which has been taken as an object of investigation. A device made of such strange components cannot, at least in principle, be ascribed any proper determination either. According to the general rules of quantum mechanics the configurations "H.E.A.D." and "T.A.I.L." of the computer can but be considered as values of an observable in turn. Therefore, the probability of each of these configurations is itself only defined relative to the interaction 
between the computer and something else; for instance between the computer and the cat or the colleague we have sent in the laboratory. If we now repeat the same reasoning a step further, for the cat (Dead or Alive) and for the colleague (aware of one of the results), we land into two well-known paradoxes of quantum mechanics: the Schrödinger's cat paradox and the Wigner's friend paradox.

Indeed, in the same way as, in the first experiment, the values of the initial probability function were propagated from one link of the measurement chain to the next one, their being relative to a possible interaction with something else is now also propagated from one link of the measurement chain to the next one. Just as a property of the screen is relative to its interaction with a cat, the biological properties of the cat are relative to its interaction with an observer, and the properties of this observer are relative to its interaction with another observer, etc. Having described the state of the strange coin by a wave function in order to express the contextuality of its determinations, one is thus progressively led to describe the state of the whole chain of measurement, including the cat and the colleague, by a wave function, in order to express the contextuality of their determinations as well.

Now, what happens if WE open the door of the lab. to see what happened in it? As previously, we see a well-defined chain of characters on the screen of the computer, we see that the cat is either dead or alive, and we hear a colleague saying that he had already seen (well before our arrival) what we are now discovering. And as previously, we must modify suddenly our tool of probabilistic prediction in order to account for our certainty of finding everything in the same configuration, if we come back in the lab. some time later. In the same way as we had to reduce the probability function of the whole measurement chain, we now have to reduce its wave function. There is a big difference between the two cases, however: whereas the ordinary probability functions could be considered as the expression of our ignorance about what effectively occurred in the lab., this is not true for the wave functions of quantum mechanics. The wave function of the measurement chain just provides a probabilistic prediction of the result of a possible (uncontrollable) interaction between it and something else. And this manifests itself through the persistent presence of a term W (for "wavering" or "wavelike effects"), in the probabilities calculated from the wave function.

But then, it seems that by entering into the lab. and becoming aware of what is going on within it, we have done something much more important than just reducing suddenly our own ignorance. We have reduced a genuine indetermination of the whole measurement chain including the cat and the colleague. We have forced it to acquire well-defined properties: the screen now really displays "T.A.I.L.", the cat is alive, and the colleague perfectly knows all this. Consciousness, and more precisely OUR consciousness, has so to speak expanded out of its natural boundaries. Far from accepting to follow the usual rules of dualism, which separate what occurs in the mind and what occurs out there, our consciousness seems to have acquired an essential role in the very process by which objects of the world acquire properties.

Yet, very few physicists, and very few philosophers as well, are prepared to accept these consequences, especially when they are formulated in such crude terms. From our initial analysis of mind and consciousness it is quite easy to understand the reason of their discomfort. Here are two entities, mind and consciousness, which were just invented to embody privacy, and which are now being ascribed the role of determining the properties of entities of the public world. Isn't there an alternative to this quite discomforting way of dealing with the quantum configuration? Actually, there are many such alternatives and I shall describe two of them.

The first one arises from the following remark. If one could at least consider that the measurement apparatuses behave almost as if they had absolute properties even if we are ignorant of them, then the reduction of their wave function would loose its specificity with respect to the reduction of a function of probability. Everything would revert to the previous crypto-dualistic scheme, since consciousness could be bracketed in the solution of problems of physics. Now, we perfectly know the conditions for probabilities to be construed as expressing our ignorance of an outcome which has already occurred. This condition is that the term $\mathrm{W}$ of interference or wave-like effects vanishes in the summation rule of quantum probabilities. Such a fast process of virtual disappearance of the interference terms (in the presence of big and 
complex objects as measuring apparatuses, or apparatuses linked to a complex environment) is "decoherence". As soon as this occurs, each element of the measurement chain, from the apparatus to the colleague, behaves approximately as if it had already acquired definite properties that we just ignore, and of which we could become aware by entering into the lab. One could thus stop at this point and say that there is no further problem since now there is no difference left between the measurement problem of quantum mechanics and the connection between classical probabilities and actual outcomes (J. Bub [2008]). Some physicists, as Bernard d'Espagnat [1994], have however pointed out that a reasoning based on decoherence does not solve the fundamental difficulty which motivated the irruption of consciouness on the stage of physics. True, the interference terms become exeedingly small after the decoherence process, but they do not disappear. Their presence prevents one in principle from considering that quantum probabilities are just expressions of ignorance, even if (at the end of the decoherence process) one can behave in practice as if they were (see H. Lyre [2003], U. Mohrhoff [2001]) for a careful analysis of this point). So, another (not necessarily antagonistic, but rather complementary) move is still needed at this stage. Everett's interpretation of quantum mechanics is often used to complement decoherence, but my feeling is that things may be sorted out in a much more economic way. The right move might just consist in unearthing the pronominal or indexical roots of the concepts of mind and consciousness.

\section{Discussion}

Let me show how this indexical "solution" (or "dissolution") of the measurement problem of quantum mechanics works. It is based on the far-reaching, and already documented, difference between a property and an observable. A property of an object is defined in the absolute, whereas an observable refers to a possible relation between the object and something else. Each time one wishes to make the relation explicit, one has to introduce the "something else" into the field of description. But then, the characterization of this something else is itself relative to a possible interaction with a third element, and so on and so forth. Isn't there a natural end to this chain of relations? Yes, there is. WE are this end. Of course, I am not trying to say that WE are unique or privileged beings in nature (this would be collective solipsism of an absurd sort), but only that WE are privileged beings for US! As soon as we establish a relation with an element of the measurement chain, this element acquires a determination relative to US. Nothing has thus to be changed in the physical description, since determinations of the measurement chain are still relative to something. But everything is different for US, since the determinations of the measurement chain are now relative to US. And a relation of which WE are one term is something quite peculiar, even if it is only peculiar ... from OUR point of view.

At this point, one might still feel perplex and wonder whether there is anything more than just a cosmetic difference between this indexical interpretation, and the good old solipsist or mentalist views. I thus still have to show where the clear-cut dividing line between these two types of conception arises. Let me recall once again that, according to the analysis of section 1., Mind has the status of a reified projection of the first-person viewpoint. In the mentalistic perpective, the fact that WE see a well-defined outcome for the experiment must accordingly be translated in terms of a property possessed by a mind, say a mental content. And this property is in turn supposed to be privately accessible to consciousness. Thus, the whole series of relations which unites the parts of the measurement chain, and which finds its expression in the use of a global wave function instead of the usual probability function, has to be broken at the point where mind and consciousness intervene. The chain of relations, and the wavelike description as well, seem to stop when absolute properties of an entity called "consciousness" come into play.

But this is no more the case if we come back to the indexical ground of the mentalistic discourse. For, in this case, no transition from relations to absolute properties is required. In the framework of a naturalizing analysis of ourselves, we can still consider ourselves as part of a chain of relations, then accepting that we partake of the description of the whole measurement chain by a wave function. And at the same time, in the framework of a phenomenological 
transcendental analysis, we are entitled to remark that since this set of relative determinations becomes at one stage relative to US, it takes for US, namely from OUR self-singled out point of view, the aspect of a well-defined result.

To sum up, at no point do we have to stop the description of the measurement chain, including ourselves, by a wave function. In order to connect this relational description with our experience and our collective agreement about a certain result, we only have to acquire a double view of ourselves: ourselves as one part among many others of the chain of relations which constitute the world, and ourselves as privileged beings ... for ourselves; ourselves as naturalized entities, and ourselves as transcendental backgrounds.

This represents a major difference with classical physics. In classical physics the simple truth that we act as situated subjects of knowledge could be bracketed, and a naturalized description of the world including ourselves taken as objects could pretend to be universal. Instead, quantum physics manifests the bounds of this attitude of all-pervasive naturalization. It makes one realize that the irreducible fact of situatedness is a necessary presupposition of objective knowledge and cannot thus be objectified itself. This, of course, was pointed out by many generations of transcendental philosophers, from Kant to Husserl and beyond ; but quantum physics leaves little room for those who want to ignore their lesson.

\section{Bibliography}

Bacciagaluppi G, Valentini A Quantum Theory at the Crossroads: Reconsidering the 1927 Solvay Conference, Cambridge University Press (To be published) 2008

Bitbol M., Physique et philosophie de l'esprit, Flammarion, 2000a

Bitbol M Physique quantique et cognition. Revue Internationale de Philosophie 54, 299-328, $2000 \mathrm{~b}$

Bitbol M, Science as if situation mattered. Phenomenology and the Cognitive Science 2002, 1 : $181-224$

Bitbol M Is Consciousness Primary ? NeuroQuantology 6, 53-72, 2008

Bub J Heisenberg's 'Umdeutung' and the information-theoretic interpretation of quantum mechanic, Conference Perspective in Physics and in Philosophy, Paris 2008

D’Espagnat B Veiled Reality, Perseus Books, 1999

Destouches-Février P L'interprétation physique de la mécanique ondulatoire et des théories quantiques, Gauthier-Villars, 1956

French S A phenomenological solution to the measurement problem? Husserl and the foundations of quantum mechanics. Studies In History and Philosophy of Science Part B: Studies In History and Philosophy of Modern Physics, 33, 467-491, 2002

Hameroff SR, Penrose R Conscious events as orchestrated spacetime selections. Journal of Consciousness Studies 3, 36-53, 1996

London F, Bauer E La Théorie de l'Observation en Mécanique Quantique, Hermann 1939

Lyre H Against Measurement. On the Concept of Information. In : Lecture Notes in Physics 517, Quantum Future From Volta and Como to the Present and Beyond, Springer, 1999

Mohrhoff U Two theories of decoherence. arXiv: quant-ph/0108002

Penrose R Shadows of the Mind, Oxford University Press 1994 
Rovelli C Relational Quantum Mechanics. International Journal of Theoretical Physics $35,1637-1678,1996$

Ryle G The Concept of Mind. Hutchinson, 1949

Schrödinger E The Present Situation in Quantum Mechanics (1935) in : Wheeler J and Zurek WH eds. Quantum Theory and Measurement, Princeton university Press, New Jersey 1983

Schrödinger E The Interpretation of Quantum Mechanics, (Bitbol M ed.) Ox Bow Press, 1995

Stapp H Mindful Universe: Quantum Mechanics and the Participating Observer Springer, 2007

Von Neumann J Mathematical Foundations of Quantum Mechanics, Princeton University Press, 1955

Wigner EP Remarks on the mind-body question in: Symmetries and Reflections, Indiana University Press 1967

Wittgenstein L Notes for lectures on 'Private experience and sense data', T.E.R. 1982 